PROCEEDINGS OF THE

AMERICAN MATHEMATICAL SOCIETY

Volume 136, Number 8, August 2008, Pages 2887-2893

S 0002-9939(08)09361-1

Article electronically published on April 14, 2008

\title{
A LOWER BOUND FOR THE DENSITY OF STATES OF THE LATTICE ANDERSON MODEL
}

\author{
PETER D. HISLOP AND PETER MÜLLER
}

(Communicated by Walter Craig)

Dedicated to Jean-Michel Combes on the occasion of his $65^{\text {th }}$ birthday

\begin{abstract}
We consider the Anderson model on the multi-dimensional cubic lattice and prove a positive lower bound on the density of states under certain conditions. For example, if the random variables are independently and identically distributed and the probability measure has a bounded Lebesgue density with compact support, and if this density is essentially bounded away from zero on its support, then we prove that the density of states is strictly positive for Lebesgue-almost every energy in the deterministic spectrum.
\end{abstract}

Wegner's estimate, originally formulated in $\mathrm{W}$ for the Anderson model on the lattice $\mathbb{Z}^{d}$, is one of the celebrated tools in the theory of random Schrödinger operators; see e.g. the recent reviews $[\mathrm{V}, \mathrm{KMe}$ or $[\mathrm{CHK}]$ for the latest developments in the case of continuum random Schrödinger operators. In its strongest form for lattice models, a Wegner estimate provides Lipschitz continuity of the integrated density of states $N(E)$. In particular, this implies that the Lebesgue derivative of $N(E)$, the density of states $n(E)$, exists as a function which is essentially bounded from above. In addition to the upper bound for the density of states, Wegner also presented an argument for a strictly positive lower bound for the density of states of the Anderson model in his original article [W]. Although insightful, Wegner's argument is not complete, as his nonzero lower bound vanishes in the macroscopic limit.

In this paper, we give a mathematical proof of a positive lower bound for the density of states of the Anderson model. For many years, efforts have been concentrated on Wegner's upper bound because this is essential for the continuity of the integrated density of states, the existence of the density of states, and for Anderson localization. Consequently, Wegner's idea to obtain a lower bound seems to have remained fairly unnoticed. The lower bound is, however, essential for Minami's proof that the energy level statistics for energies in the strong localization regime is Poissonian Min. Minami fixes an energy $E$ in the region of complete localization at which the fractional moment bounds of Aizenman and Molchanov [AM hold. He assumes that $n(E)>0$. Minami then proves that the rescaled local eigenvalue level spacing measure $\mathrm{d} \mu_{L}(x)=\sum_{j} \delta\left(L^{d}\left(\varepsilon_{j}(L)-E\right)-x\right) \mathrm{d} x$, for the finite-volume

Received by the editors May 11, 2007.

2000 Mathematics Subject Classification. Primary 47B80, 35P15, 81Q10.

Key words and phrases. Random Schrödinger operators, integrated density of states, Wegner estimate, lower bound. 
Hamiltonian (see below for the definition) with eigenvalues $\varepsilon_{j}(L)$, converges in the macroscopic limit to a Poisson distribution with density given by $n(E)$. Here, we prove the positivity of the density of states at almost every energy in the deterministic spectrum. We mention that Molchanov [Mol] studied the same question of energy-level statistics for the one-dimensional Russian school model. In his paper, he also proves the positivity of the density of states for that model using completely different methods.

The Anderson model is given by the discrete random Schrödinger operator $H$ on a probability space $(\Omega, \mathbb{P})$ whose realizations $H^{(\omega)}:=\mathcal{L}+V^{(\omega)}, \omega \in \Omega$, act as

$$
\left(H^{(\omega)} \varphi\right)(x)=(\mathcal{L} \varphi)(x)+\omega_{x} \varphi(x)
$$

for all $x \in \mathbb{Z}^{d}$ on a dense domain of $\varphi \in \ell^{2}\left(\mathbb{Z}^{d}\right)$. Here, the discrete Laplacian $\mathcal{L}$ is defined as $(\mathcal{L} \varphi)(x):=\sum_{y \in \mathbb{Z}^{d}:|x-y|=1} \varphi(y)$, and has purely absolutely continuous spectrum $\sigma(\mathcal{L})=[-2 d, 2 d]$. The random potential $V^{(\omega)}$ consists of a family $\left\{\omega_{x}\right\}_{x \in \mathbb{Z}^{d}}$ of independent, identically distributed real-valued random variables on $\Omega$.

The Schrödinger operator $H$ of the Anderson model is known $[\mathrm{CL}, \mathrm{PF}$ to be almost surely essentially self-adjoint on the dense subspace $\left\{\varphi \in \ell^{2}\left(\mathbb{Z}^{d}\right)\right.$ : $\operatorname{supp} \varphi$ compact $\}$. Moreover, $H$ is ergodic with respect to lattice translations. To define the integrated density of states, we consider finite volumes $\Lambda \subset \mathbb{Z}^{d}$ and the Dirichlet restriction $H_{\Lambda}^{(\omega)}:=\mathcal{L}_{\Lambda}+V_{\Lambda}^{(\omega)}$ of $H$ to the finite-dimensional Hilbert space $\ell^{2}(\Lambda)$, where $V_{\Lambda}^{(\omega)}$ is the restriction of $V^{(\omega)}$ to $\Lambda$ and

$$
\left(\mathcal{L}_{\Lambda} \varphi\right)(x):=\sum_{y \in \Lambda:|x-y|=1} \varphi(y)+\varphi(x)\left(\sum_{y \notin \Lambda:|x-y|=1} 1\right)
$$

for all $x \in \Lambda$ and all $\varphi \in \ell^{2}(\Lambda)$. Note that the rightmost term in the above definition of the Dirichlet Laplacian $\mathcal{L}_{\Lambda}$ ensures the Dirichlet decoupling estimate $\mathcal{L}_{\Lambda_{1} \cup \Lambda_{2}} \leq \mathcal{L}_{\Lambda_{1}} \oplus \mathcal{L}_{\Lambda_{2}}$; see also [S, KiMü]. We write $\operatorname{tr}_{\Lambda}$ for the trace on $\ell^{2}(\Lambda)$ and let $\chi_{B}$ stand for the indicator function of some set $B \subset \mathbb{R}$. Then ergodicity implies that the integrated density of states $E \in \mathbb{R} \mapsto N(E)$ is given by the non-random limit

$$
N(E)=\lim _{\Lambda \uparrow \mathbb{Z}^{d}}\left[\frac{1}{|\Lambda|} \operatorname{tr}_{\Lambda}\left(\chi_{]-\infty, E]}\left(H_{\Lambda}^{(\omega)}\right)\right)\right]
$$

along a sequence of expanding cubes $\Lambda \subset \mathbb{Z}^{d}$. Equation (3) holds for all $E \in \mathbb{R}$ that are continuity points of $N(E)$ and all $\omega \in \Omega$ except for a $\mathbb{P}$-null set, which can be chosen uniformly with respect to the aforementioned values of $E$.

If the single-site distribution of, say, $\omega_{0}$ happens to be absolutely continuous with respect to Lebesgue measure and if its Lebesgue density $\rho$ satisfies the additional assumption

$$
\rho_{\max }:=\operatorname{ess}_{w \in \mathbb{R}}\{\rho(w)\}<\infty,
$$

then, according to Wegner [W], the integrated density of states $N(E)$ is Lipschitz continuous, hence absolutely continuous, and the Lebesgue derivative of $N(E)$, the density of states,

$$
E \in \mathbb{R} \mapsto n(E):=\mathrm{d} N(E) / \mathrm{d} E
$$


obeys the estimate $n(E) \leq \rho_{\max }$ for Lebesgue-almost all $E \in \mathbb{R}$. Another consequence of ergodicity of the Schrödinger operator $H$ is that there is a closed set $\Sigma \subset \mathbb{R}$ such that $\sigma\left(H^{(\omega)}\right)=\Sigma$ with probability one. This set, called the deterministic spectrum of $H$, is given by $\Sigma=[-2 d, 2 d]+\operatorname{supp} \rho$, for the model in (1).

We will not assume (4) for the validity of the lower bound for $n(E)$.

Theorem 1. Let $H$ be the random Schrödinger operator (1) of the Anderson model. Assume that the single-site distribution of $\omega_{0}$ is absolutely continuous with respect to Lebesgue measure and that its Lebesgue density $\rho$ is essentially bounded away from zero on some interval $\left[W_{-}, W_{+}\right]$in the sense that

$$
\rho_{\min }:=\underset{w \in\left[W_{-}, W_{+}\right]}{\operatorname{essinf}}\{\rho(w)\}>0
$$

for some $-\infty<W_{-}<W_{+}<\infty$. Assume further that the integrated density of states $N(E)$ is an absolutely continuous function with Lebesgue derivative $n(E)$ as in (5). Then, for every $\delta>0$ (small enough) there exists a strictly positive constant $C_{\delta}$ such that

$$
n(E) \geq C_{\delta}>0,
$$

for Lebesgue-almost all $E \in\left[-2 d+W_{-}+\delta, 2 d+W_{+}-\delta\right]$.

Remark 1. The theorem can be generalized in a straightforward manner to incorporate general bounded, self-adjoint and $\mathbb{Z}^{d}$-translation invariant unperturbed operators $H_{0}$ instead of $\mathcal{L}$. In this case, (7) holds for all $E \in \sigma\left(H_{0}\right)+\left[W_{-}+\delta, W_{+}-\delta\right]$.

Remark 2. The lower bound constant $C_{\delta}>0$ can be expressed in terms of the integrated density of states $N^{(0)}(E)$ for the unperturbed operator $\mathcal{L}$. For any $\delta>0$ small enough, we cover the interval $\left[-2 d+W_{-}+\delta, 2 d+W_{+}-\delta\right]$ by finitely many intervals $I_{j}=\left[E_{j}-\delta, E_{j}+\delta\right]$ of width $2 \delta$ and centered at $E_{j}$. We can take $C_{\delta}$ to be

$$
C_{\delta}=\min _{j}\left\{\frac{\left(\delta \rho_{\min }\right)^{\alpha_{E_{j}}}}{2 \delta}\left[N^{(0)}\left(E_{j}-W_{-}-2 \delta\right)-N^{(0)}\left(E_{j}-W_{+}+2 \delta\right)\right]\right\},
$$

where the finite positive constants $\alpha_{E_{j}}>0$ are defined in the proof of Theorem 1] The difference of the integrated densities of states for $\mathcal{L}$ on the right of (을 is strictly positive; see the end of the proof of Theorem 1 .

In Theorem 1, we do not require that $\rho$ be essentially bounded as in (4), that the support be bounded, or that $\left[W_{-}, W_{+}\right]$be the entire support of $\rho$. However, if we add the latter two hypotheses, we obtain the following special case of Theorem 1 .

Corollary 1. Under the hypotheses of Theorem 1 and the additional condition that (6) holds on the entire support of $\rho$, i.e. $\rho(w)=0$ for almost every $w \in \mathbb{R} \backslash\left[W_{-}, W_{+}\right]$, the density of states is strictly positive Lebesgue-almost everywhere on $\Sigma$.

Remark 3. After we completed this work, we learned that a similar result is contained in Frank Jeske's unpublished Ph.D. thesis J], which was supervised by Werner Kirsch. We thank Ivan Veselić for informing us about the existence of J].

Remark 4. The question arises naturally as to whether a similar lower bound for the density of states $n$ does also hold in the case of continuum random Schrödinger operators, that is, Schrödinger operators on $L^{2}\left(\mathbb{R}^{d}\right)$. For $d=1$ and for alloy-type random potentials with suitably well-behaved single-site potentials, the answer is 
affirmative. The argument proceeds as in the discrete case with some obvious modifications that are well known from proofs of upper Wegner estimates for continuum models. The key point is that the finite-rank-perturbation argument, which allows us to proceed from (15) to (16) below, is still valid in the one-dimensional continuum case. Indeed, suppose we have two Schrödinger operators on an interval that differ only by a boundary condition (Dirichlet versus none, say) that is imposed at an interior point of the interval. Then it is well known from e.g. the theory of point interactions that these two Schrödinger operators differ by a rank-2 perturbation. However, for $d \geq 2$, different boundary conditions along a finite hypersurface $S$ give rise to an infinite-rank perturbation. Thus, in the case $d \geq 2$ one needs an alternative argument as to why different boundary conditions along $S$ for Schrödinger operators in a finite volume $\Lambda$ (with $S$ in the interior of $\Lambda$ ) lead to eigenvalue counting functions that differ by a term proportional to the area of $S$. Furthermore, this error term would be required to remain bounded as $\Lambda \uparrow \mathbb{R}^{d}$. But this is a delicate issue in view of [K1, K2].

Proof of Theorem 1. The proof follows Wegner's arguments [W], except that we introduce a partition of the finite volume into cubes of large but fixed size. This allows us to get a non-trivial result in the macroscopic limit $L \rightarrow \infty$, a problem seemingly ignored in $\overline{\mathrm{W}}$. A similar partitioning strategy was used in the proof of a (upper) Wegner estimate for continuum random Schrödinger operators by spectral averaging [CH]; see also [FHLM, HLMW] for the case of Gaussian or other types of unbounded random potentials.

1. Let $E_{1}, E_{2} \in \mathbb{R}$ be such that $E_{2}-E_{1}>\varepsilon$ for some $\varepsilon>0$. We consider a sequence of expanding cubes $\Lambda_{L}$ in $\mathbb{Z}^{d}$ with volume $\left|\Lambda_{L}\right|=L^{d}$. Finally, we pick a smooth, monotone increasing switch function $f_{\varepsilon} \in C^{1}(\mathbb{R})$ such that $f_{\varepsilon}(\lambda)=0$ for all $\lambda \leq 0$ and $f_{\varepsilon}(\lambda)=1$ for all $\lambda \geq \varepsilon$. We let $\mathbb{E}$ denote the expectation associated with the probability measure $\mathbb{P}$, and we write $F_{\varepsilon, L}(\lambda, \omega):=\operatorname{tr}_{\Lambda_{L}} f_{\varepsilon}\left(\lambda-H_{\Lambda_{L}}^{(\omega)}\right)$. Then we have

$$
\begin{aligned}
N\left(E_{2}\right)-N\left(E_{1}\right) & \geq \lim _{L \rightarrow \infty}\left\{\frac{1}{L^{d}} \mathbb{E}\left[\operatorname{tr}_{\Lambda_{L}}\left(f_{\varepsilon}\left(E_{2}-H_{\Lambda_{L}}\right)-f_{\varepsilon}\left(E_{1}+\varepsilon-H_{\Lambda_{L}}\right)\right)\right]\right\} \\
& =\lim _{L \rightarrow \infty}\left\{\frac{1}{L^{d}} \int_{E_{1}+\varepsilon}^{E_{2}} \mathrm{~d} \lambda \mathbb{E}\left[\frac{\partial}{\partial \lambda} F_{\varepsilon, L}(\lambda, \cdot)\right]\right\} .
\end{aligned}
$$

The quantity $F_{\varepsilon, L}(\lambda, \omega)$ depends on $\lambda$ and $\omega$ only through the differences $\left\{\omega_{x}-\lambda\right\}_{x \in \Lambda_{L}}$, and it is a monotone decreasing function in each of those differences. We partition the cube $\Lambda_{L}$ into $(L / \ell)^{d}$ smaller cubes $\Gamma_{j}$ of the same (fixed) volume $\ell^{d}$. We consider only those big cubes $\Lambda_{L}$ for which such a partition is possible. We will take $L \rightarrow \infty$ and $\ell$ large but finite. Therefore we get

$$
\frac{\partial}{\partial \lambda} F_{\varepsilon, L}(\lambda, \omega)=-\sum_{j=1}^{(L / \ell)^{d}} \sum_{x \in \Gamma_{j}} \frac{\partial}{\partial \omega_{x}} F_{\varepsilon, L}(\lambda, \omega)
$$

for all $\lambda \in \mathbb{R}$ and all $\omega \in \Omega$.

2. We conclude from (9) and (10) that

$$
N\left(E_{2}\right)-N\left(E_{1}\right) \geq \rho_{\min }^{\ell^{d}} \lim _{L \rightarrow \infty}\left\{\frac{1}{(L / \ell)^{d}} \sum_{j=1}^{(L / \ell)^{d}} \mathbb{E}_{\Gamma_{j}^{c}}\left[\int_{E_{1}+\varepsilon}^{E_{2}} \mathrm{~d} \lambda G_{j}(\lambda, \cdot)\right]\right\}
$$


with

$$
G_{j}\left(\lambda, \omega_{\Gamma_{j}^{c}}\right):=\frac{1}{\ell^{d}} \int_{\left[W_{-}, W_{+}\right]^{\ell^{d}}}\left(\prod_{y \in \Gamma_{j}} \mathrm{~d} \omega_{y}\right) \sum_{x \in \Gamma_{j}}\left(-\frac{\partial}{\partial \omega_{x}}\right) F_{\varepsilon, L}(\lambda, \omega) .
$$

Here $\Gamma_{j}^{c}:=\mathbb{Z}^{d} \backslash \Gamma_{j}$ denotes the complement of $\Gamma_{j}$, and (in slight abuse of notation) we have written $\omega=$ : $\left(\omega_{\Gamma_{j}}, \omega_{\Gamma_{j}^{c}}\right)$, where $\omega_{\Gamma_{j}}:=\left(\omega_{x}\right)_{x \in \Gamma_{j}}$. The partial disorder average $\mathbb{E}_{\Gamma_{j}^{c}}$ in (12) extends only over the coupling constants $\omega_{\Gamma_{j}^{c}}$.

3. Following Wegner [W], we are going to perform a change of variables in (12) from $\omega_{\Gamma_{j}}$ to $\eta$ : we fix an arbitrary point $x_{j} \in \Gamma_{j}$ and set $\eta_{x_{j}}:=\omega_{x_{j}}$ and $\eta_{y}:=\omega_{y}-\omega_{x_{j}}$ for all $y \in \Gamma_{j} \backslash\left\{x_{j}\right\}$. The Jacobian associated with this change of variables is 1 , whence

$$
\begin{aligned}
\left.G_{j}\left(\lambda, \omega_{\Gamma_{j}^{c}}\right)=\frac{1}{\ell^{d}} \int_{\left[W_{-}, W_{+}\right]} \mathrm{d} \eta_{x_{j}} \int_{\left[W_{-}\right.}-\eta_{x_{j}}, W_{+}-\eta_{x_{j}}\right]^{\ell^{d}-1} & \left(\prod_{y \in \Gamma_{j} \backslash\left\{x_{j}\right\}} \mathrm{d} \eta_{y}\right) \\
& \times\left(-\frac{\partial}{\partial \eta_{x_{j}}}\right) F_{\varepsilon, L}\left(\lambda,\left(\omega_{\Gamma_{j}}(\eta), \omega_{\Gamma_{j}^{c}}\right)\right) .
\end{aligned}
$$

Now, fix $\delta \in] 0,\left(W_{+}-W_{-}\right) / 4[$. One obtains a lower bound for (13) by first restricting the integration over $\eta_{x_{j}}$ to $\left[W_{-}+\delta / 2, W_{+}-\delta / 2\right]$ and then restricting the integration over $\eta_{y}$ to $[-\delta / 2, \delta / 2]$, for all $y \in \Gamma_{j} \backslash\left\{x_{j}\right\}$. This gives

$$
\begin{aligned}
G_{j}\left(\lambda, \omega_{\Gamma_{j}^{c}}\right) \geq \frac{1}{\ell^{d}} \int_{[-\delta / 2, \delta / 2]^{\ell^{d}-1}}\left(\prod_{y \in \Gamma_{j} \backslash\left\{x_{j}\right\}} \mathrm{d} \eta_{y}\right)[ & {\left[F_{\varepsilon, L}\left(\lambda,\left(\omega_{\Gamma_{j}}\left(\eta^{-}\right), \omega_{\Gamma_{j}^{c}}\right)\right)\right.} \\
& \left.-F_{\varepsilon, L}\left(\lambda,\left(\omega_{\Gamma_{j}}\left(\eta^{+}\right), \omega_{\Gamma_{j}^{c}}\right)\right)\right]
\end{aligned}
$$

with $\eta^{ \pm}:=\left(W_{ \pm} \mp \delta / 2,\left(\eta_{y}\right)_{y \in \Gamma_{j} \backslash\left\{x_{j}\right\}}\right)$. Note that in (14) one has $\left(\omega_{\Gamma_{j}}\left(\eta^{-}\right)\right)_{x} \leq$ $W_{-}+\delta$ and $\left(\omega_{\Gamma_{j}}\left(\eta^{+}\right)\right)_{x} \geq W_{+}-\delta$ for all $x \in \Gamma_{j}$. Since $F_{\varepsilon, L}$ is a decreasing function in each $\omega_{x}$, we arrive at

$$
G_{j}\left(\lambda, \omega_{\Gamma_{j}^{c}}\right) \geq \frac{\delta^{\ell^{d}-1}}{\ell^{d}}\left[F_{\varepsilon, L}\left(\lambda,\left(\omega_{\Gamma_{j}}^{-}, \omega_{\Gamma_{j}^{c}}\right)\right)-F_{\varepsilon, L}\left(\lambda,\left(\omega_{\Gamma_{j}}^{+}, \omega_{\Gamma_{j}^{c}}\right)\right)\right]
$$

with spatially constant couplings $\omega_{\Gamma_{j}}^{ \pm}:=\left(W_{ \pm} \mp \delta\right)_{x \in \Gamma_{j}}$ inside the small cube $\Gamma_{j}$.

4. Next, we will use a Dirichlet decoupling of the small cube $\Gamma_{j}$. In the first (i.e. the positive) term on the right-hand side of (15), this can be done straight away, because $H_{\Lambda_{L}} \leq H_{\Gamma_{j}} \oplus H_{\Lambda_{L} \backslash \Gamma_{j}}$. To do the replacement in the second (i.e. the negative) term, one has to take into account the error that arises from introducing the additional Dirichlet boundary condition along $\partial \Gamma_{j} \backslash \partial \Lambda_{L}$. But this is a perturbation of $\operatorname{rank} \mathcal{O}\left(\ell^{d-1}\right)$, and it is independent of the coupling constants. Furthermore, recall that $0 \leq f_{\varepsilon} \leq 1$. Thus there is a constant $\left.D \in\right] 0, \infty$, which depends only on $d$, such that

$$
\begin{gathered}
G_{j}\left(\lambda, \omega_{\Gamma_{j}^{c}}\right) \geq \frac{\delta^{\ell^{d}-1}}{\ell^{d}}\left\{\operatorname{tr}_{\Gamma_{j}}\left[f_{\varepsilon}\left(\lambda-W_{-}-\delta-\mathcal{L}_{\Gamma_{j}}\right)-f_{\varepsilon}\left(\lambda-W_{+}+\delta-\mathcal{L}_{\Gamma_{j}}\right)\right]\right. \\
\left.-D \ell^{d-1}\right\} \\
\geq \frac{\delta^{\ell^{d}-1}}{\ell^{d}}\left\{\begin{array}{c}
\operatorname{tr}_{\Gamma_{j}}\left[f_{\varepsilon}\left(E_{1}-W_{-}-\delta-\mathcal{L}_{\Gamma_{j}}\right)-f_{\varepsilon}\left(E_{2}-W_{+}+\delta-\mathcal{L}_{\Gamma_{j}}\right)\right] \\
\left.-D \ell^{d-1}\right\}
\end{array}\right.
\end{gathered}
$$


for all $\lambda \in\left[E_{1}, E_{2}\right]$ and all $\omega_{\Gamma_{j}^{c}}$. The contributions from $H_{\Lambda_{L} \backslash \Gamma_{j}}$ have canceled, so the right side in (16) is independent of $L$. Inserting (16) into (11) and taking the limit $\varepsilon \downarrow 0$, we arrive at the estimate

$$
\frac{N\left(E_{2}\right)-N\left(E_{1}\right)}{E_{2}-E_{1}} \geq \frac{\left(\delta \rho_{\min }\right)^{\ell^{d}}}{\delta}\left(K_{\ell}\left(E_{1}, E_{2}\right)-D / \ell\right)
$$

for the difference quotient of the integrated density of states of $H$. The lower bound in (17) is expressed in terms of the difference

$$
K_{\ell}\left(E_{1}, E_{2}\right):=N_{\Lambda_{\ell}}^{(0)}\left(E_{1}-W_{-}-\delta\right)-N_{\Lambda_{\ell}}^{(0)}\left(E_{2}-W_{+}+\delta\right)
$$

of the Dirichlet finite-volume approximation $N_{\Lambda_{\ell}}^{(0)}(\lambda):=\ell^{-d} \operatorname{tr}_{\Lambda_{\ell}} \chi_{]-\infty, \lambda]}\left(\mathcal{L}_{\Lambda_{\ell}}\right)$ for the integrated density of states of the free Laplacian $\mathcal{L}$.

5. By hypothesis we know that $N(E)$ is absolutely continuous with respect to Lebesgue measure. Hence, we can take the monotone limit $E_{2} \downarrow E_{1}=: E$ in (17) and obtain

$$
n(E) \geq \frac{\left(\delta \rho_{\min }\right)^{\ell^{d}}}{\delta}\left(K_{\ell}(E, E)-D / \ell\right)
$$

for Lebesgue-almost all $E \in \mathbb{R}$. We now fix $E_{0} \in \mathbb{R}$, and observe that $K_{\ell}(E, E) \geq$ $K_{\ell}\left(E_{0}-\delta, E_{0}+\delta\right)=: K_{\ell}\left(E_{0}\right)$, for all $E \in\left[E_{0}-\delta, E_{0}+\delta\right]$. We next note that

$$
K\left(E_{0}\right):=\lim _{\ell \rightarrow \infty} K_{\ell}\left(E_{0}\right)=N^{(0)}\left(E_{0}-W_{-}-2 \delta\right)-N^{(0)}\left(E_{0}-W_{+}+2 \delta\right)
$$

exists, where $N^{(0)}(\lambda):=\lim _{\ell \rightarrow \infty} N_{\Lambda_{\ell}}^{(0)}(\lambda)=\left\langle\delta_{0}, \chi_{]-\infty, \lambda]}(\mathcal{L}) \delta_{0}\right\rangle$. It is important to observe that $E_{0}-W_{-}-2 \delta>E_{0}-W_{+}+2 \delta$, since $0<\delta<\left(W_{+}-W_{-}\right) / 4$, and that for all $\left.E_{0} \in\right]-2 d+W_{-}+2 \delta, 2 d+W_{+}-2 \delta\left[\right.$, we have $-2 d<E_{0}-W_{-}-2 \delta<$ $2 d+\left(W_{+}-W_{-}\right)-4 \delta$, and $-2 d-\left[\left(W_{+}-W_{-}\right)-4 \delta\right]<E_{0}-W_{+}+2 \delta<2 d$. Specifically, we have $-2 d<E_{0}-W_{-}-2 \delta$ and if $E_{0}-W_{-}-2 \delta>2 d$, then the other energy satisfies $E_{0}-W_{+}+2 \delta<2 d$. Consequently, $K\left(E_{0}\right)$ is strictly positive for $E_{0}$ on the specified range since

$$
N^{(0)}\left(\lambda_{2}\right)-N^{(0)}\left(\lambda_{1}\right)>0
$$

whenever $\lambda_{1}<\lambda_{2}$, and at least one of the $\lambda_{j}$ 's lies in the interior of $\sigma(\mathcal{L})=[-2 d, 2 d]$. Thus, there exists a finite length $\ell_{E_{0}}$ such that

$$
K_{\ell_{E_{0}}}(E, E)-D / \ell_{E_{0}} \geq K\left(E_{0}\right) / 2 \quad \text { for all } E \in\left[E_{0}-\delta, E_{0}+\delta\right] .
$$

The theorem follows from (19), (22) and by covering the interval $]-2 d+W_{-}+$ $\delta, 2 d+W_{+}-\delta[$ by a finite number of small intervals of length $2 \delta$.

\section{ACKNOWLEDGements}

The authors thank François Germinet for his kind hospitality at the Université de Cergy-Pontoise and Abel Klein for several discussions.

\section{REFERENCES}

[AM] M. Aizenman, S. Molchanov, Localization at large disorder and extreme energies: An elementary derivation, Commun. Math. Phys. 157, 245-278, 1993. MR.1244867|(95a:82052)

[CL] R. Carmona, J. Lacroix, Spectral theory of random Schrödinger operators, Birkhäuser, Boston, 1990. MR1102675 (92k:47143)

[CH] J.-M. Combes, P. D. Hislop, Localization for some continuous, random Hamiltonians in d-dimensions, J. Funct. Anal. 124, 149-180, 1994. MR.1284608 (95g:82047) 
[CHK] J.-M. Combes, P. D. Hislop, F. Klopp, An optimal Wegner estimate and its application to the global continuity of the integrated density of states for random Schrödinger operators, Duke Math. J. 140, no. 3, 469-498, 2007. MR2362242

[FHLM] W. Fischer, T. Hupfer, H. Leschke, P. Müller, Existence of the density of states for multi-dimensional continuum Schrödinger operators with Gaussian random potentials, Commun. Math. Phys. 190, 133-141, 1997. MR1484550 (99b:82049)

[HLMW] T. Hupfer, H. Leschke, P. Müller, S. Warzel, The absolute continuity of the integrated density of states for magnetic Schrödinger operators with certain unbounded random potentials, Commun. Math. Phys. 221, 229-254, 2001. MR1845322 (2002i:82052)

[J] F. Jeske, Über lokale Positivität der Zustandsdichte zufälliger Schrödinger-Operatoren, Ph.D. thesis, Ruhr-Universität Bochum, Germany, 1992 [in German].

[K1] W. Kirsch, Small perturbations and the eigenvalues of the Laplacian on large bounded domains, Proc. Amer. Math. Soc. 101, 509-512, 1987. MR908658 (88k:35152)

[K2] W. Kirsch, The stability of the density of states of Schrödinger operator under very small perturbations, Integral Equations Operator Theory 12, 383-391, 1989. MR.998279 (90f:47071)

[KMe] W. Kirsch, B. Metzger, The integrated density of states for random Schrödinger operators. In: Spectral Theory and Mathematical Physics: A Festschrift in Honor of Barry Simon's 60th Birthday, Part 2, Proc. Symp. Pure Math., vol. 76, 649-696, Amer. Math. Soc., Providence, RI, 2007. MR2307751

[KiMü] W. Kirsch, P. Müller, Spectral properties of the Laplacian on bond-percolation graphs, Math. Z. 252, 899-916, 2006. MR2206633 (2007c:60100)

[Min] N. Minami, Local fluctuation of the spectrum of a multidimensional Anderson tight binding model, Commun. Math. Phys. 177, 709-725, 1996. MR1385082 (97d:82046)

[Mol] S. A. Molčanov, The local structure of the spectrum of the one-dimensional Schrödinger operator, Commun. Math. Phys. 78, 429-446, 1980/81. MR603503 (82d:35076)

[PF] L. Pastur, A. Figotin, Spectra of random and almost-periodic operators, Springer, Berlin, 1992. MR1223779 (94h:47068)

[S] B. Simon, Lifschitz tails for the Anderson model, J. Stat. Phys. 38, 65-76, 1985. MR784931 (86h:82053)

[V] I. Veselić, Integrated density of states and Wegner estimates for random Schrödinger operators. In: Spectral theory of Schrödinger operators, Contemp. Math., vol. 340, 97183, Amer. Math. Soc., Providence, RI, 2004. MR2051995 (2005b:82048)

[W] F. Wegner, Bounds on the density of states in disordered systems, Z. Phys. B 44, 9-15, 1981. MR639135 (83b:82060)

Department of Mathematics, University of Kentucky, Lexington, Kentucky 405060027

E-mail address: hislop@ms.uky.edu

Institut für Theoretische Physik, Georg-August-Universität Göttingen, FriedrichHund-Platz 1, 37077 Göttingen, Germany

Current address: Mathematisches Institut Ludwig-Maximilians-Universität, Theresienstr. 39, 80333, München, Germany

E-mail address: peter.mueller@physik.uni-goe.de 